\title{
COGNITIVE AND AFFECTIVE ALTERATIONS IN YOUNG ADULT RATS AND THEIR MALE OFFSPRING FOLLOWING EXPOSURE TO SEVOFLURANE ANESTHESIA
}

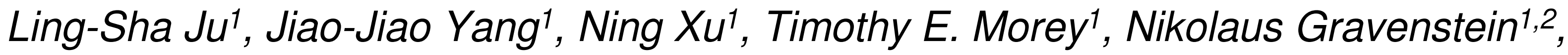
Christoph N. Seubert ${ }^{1}$, Barry Setlow ${ }^{2,3}$, and Anatoly E. Martynyuk ${ }^{1,2}$

Department of Anesthesiology ${ }^{1}$, the McKnight Brain Institute ${ }^{2}$, and Department of Psychiatry $^{3}$, University of Florida College of Medicine, Gainesville, FL.

Objectives: Investigate intergenerational effects of young adult anesthesia with sevoflurane.

Background and aims: Each year millions of patients have surgeries under general anesthesia. Anesthetic exposure in early childhood or old age is linked to neurocognitive deficiencies. The adverse effects of anesthetic exposure in young adults during their prime reproductive period remain largely unexplored.

Materials and methods: The postnatal day 56 rats (generation 1, G1) were sevoflurane anesthetized on 3 alternating days and mated 25 days later to produce offspring (G2).

Results: The G1 males and females had elevated systemic corticosterone $1 \mathrm{~h}$ after the last sevoflurane exposure. Only G1 males developed persistent neurobehavioral deficiencies, exaggerated hypothalamic-pituitary-adrenal (HPA) axis responses to restraint. Changes in hypothalamic-pituitarytesticular (HPT) axis functioning and expression of hypothalamic aromatase and estrogen receptors were consistent with a role for systemic testosterone/brain estradiol in G1 sex-specific effects of sevoflurane. Only the male offspring of exposed parents exhibited neurobehavioral deficiencies, but had unaltered HPA and HPT axis functioning. Finally, downregulated the $\mathrm{K}^{+-} \mathrm{Cl}^{-}$co-transporter KCC2 gene (Kcc2) expression in $\mathrm{G} 1$ and $\mathrm{G} 2$ male hypothalamus and hippocampus, and hyper-methylated Kcc2 promoter in G1 sperm and ovary and G2 male hypothalamus and hippocampus support the involvement of epigenetic mechanisms in sevoflurane's intergenerational effects.

Conclusions: Adult sevoflurane exposure affects brain development in male offspring by epigenetically reprograming both parental germ cells, while induces neuroendocrine and behavioral abnormalities only in exposed males.
A

G1 Sperm
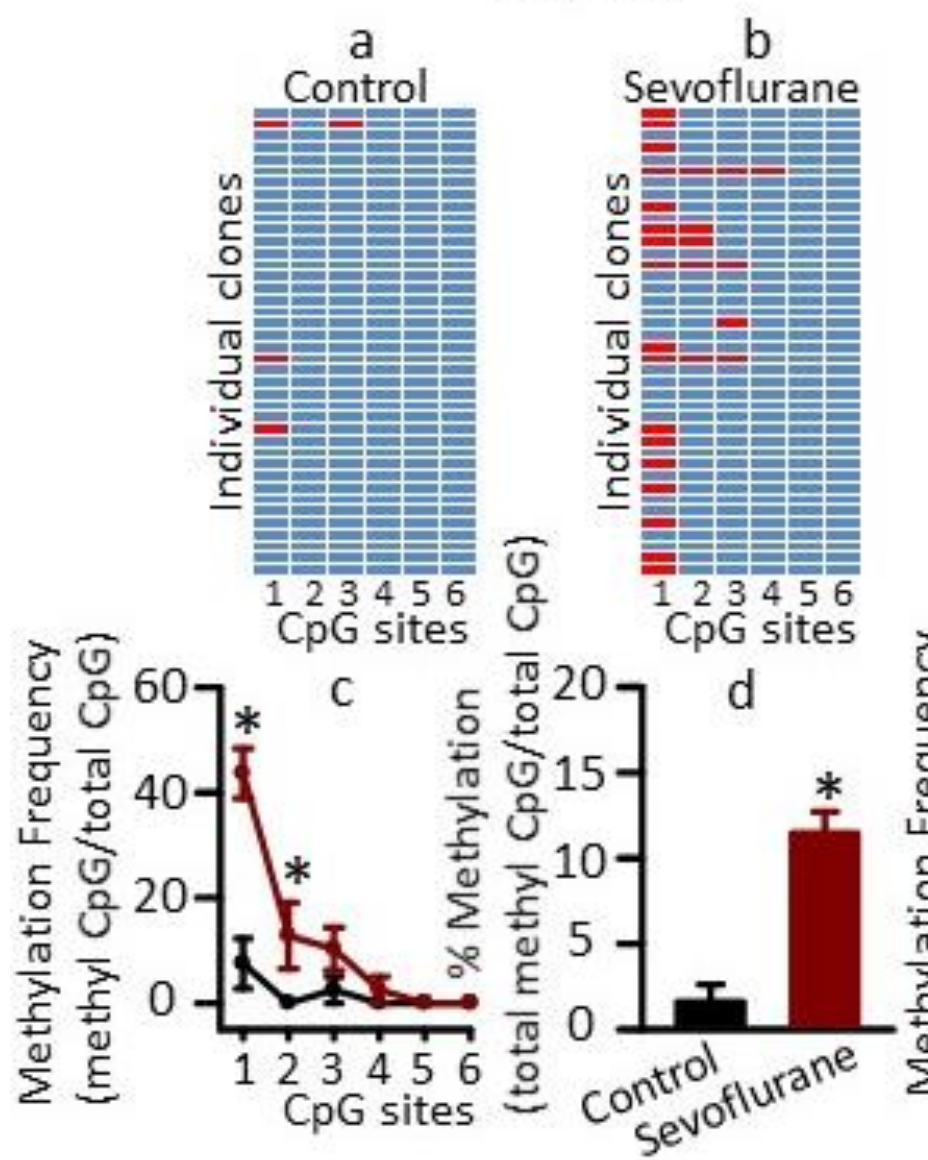

C

G2 Male Hypothalamus
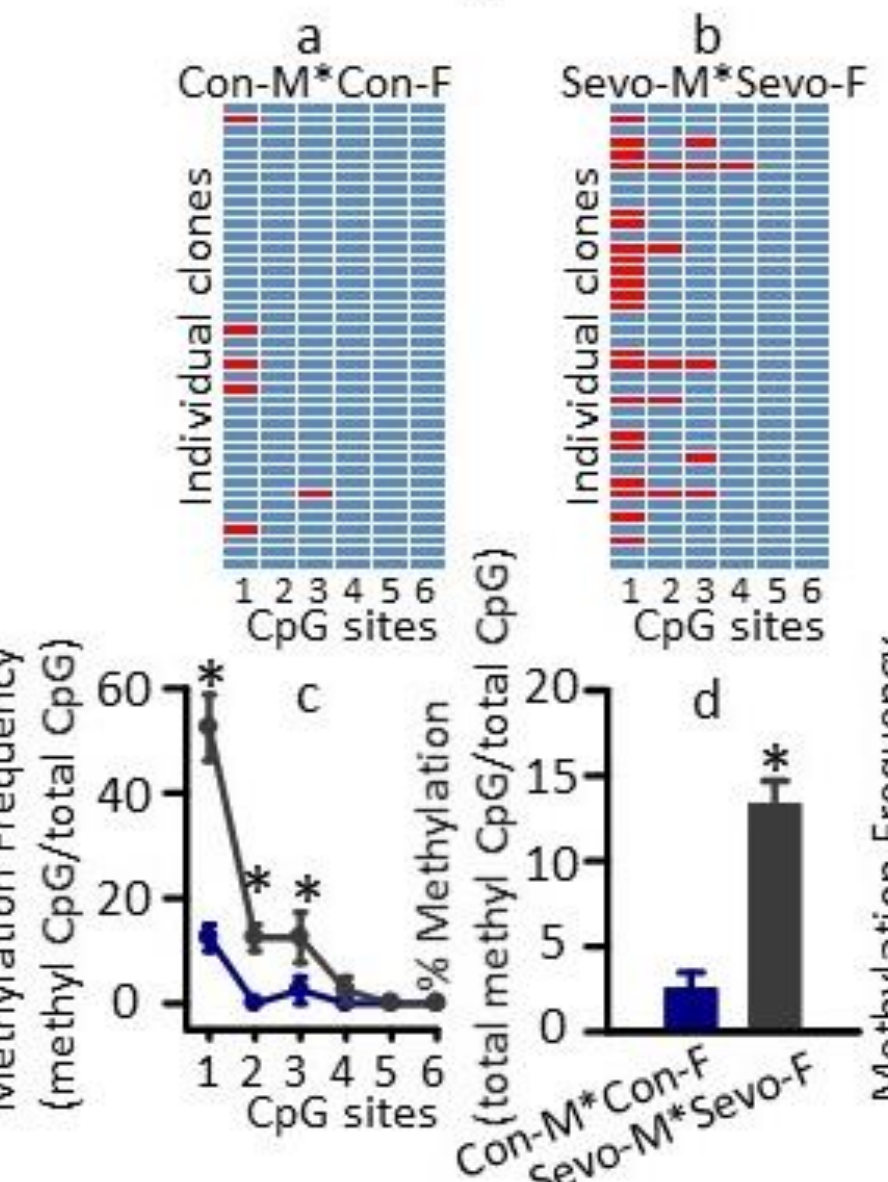
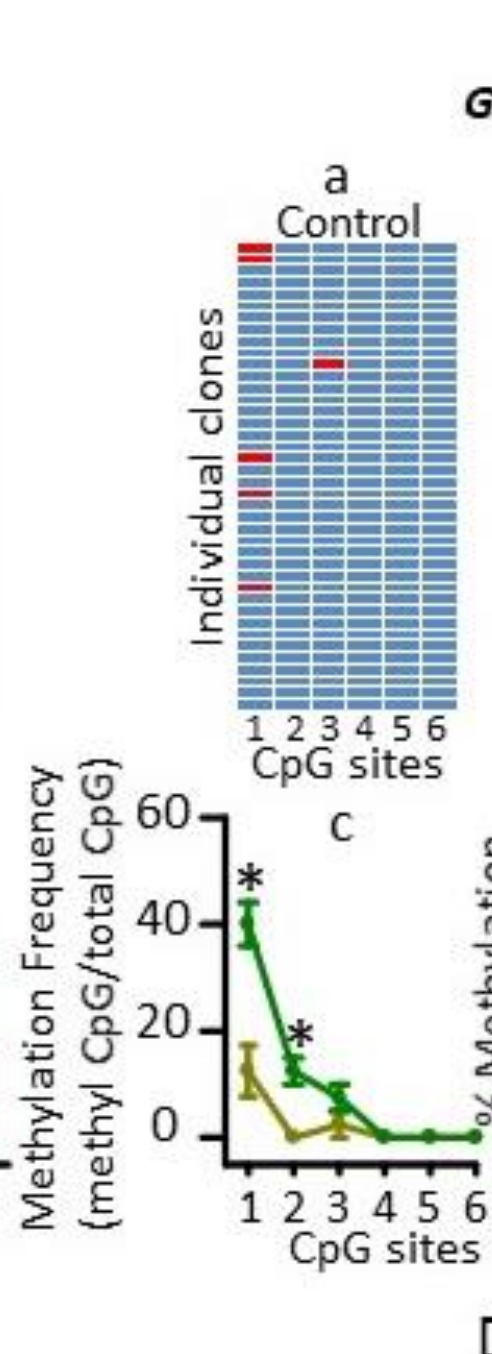

$B$ G1 Ovary

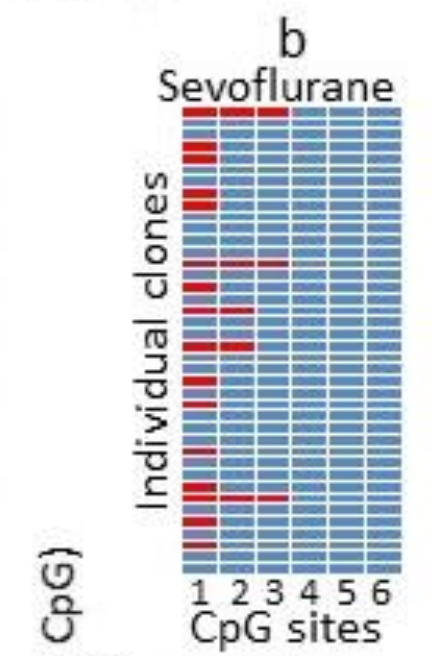
CPG sites oै 123456
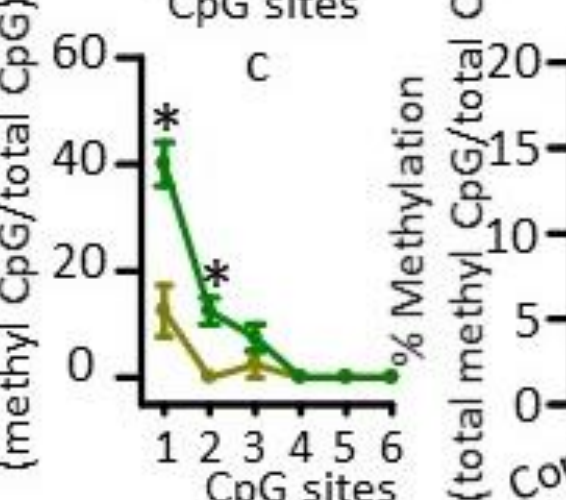

d

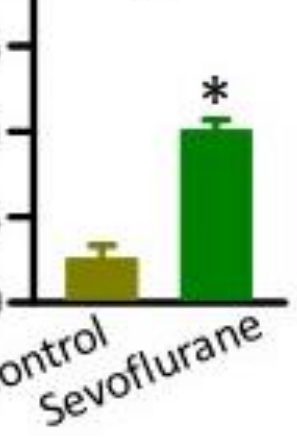

D

G2 Male Hippocampus

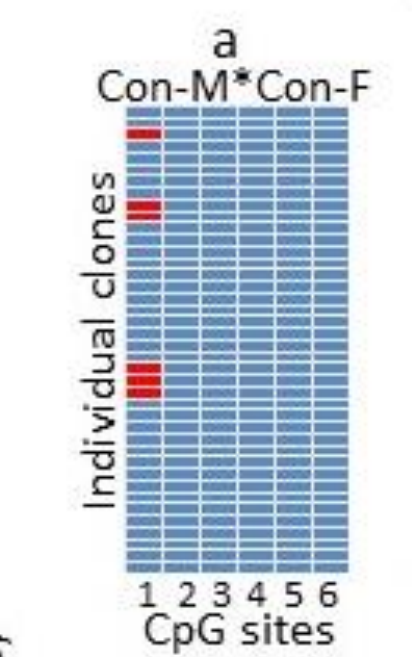

Sevo-M*Sevo-F
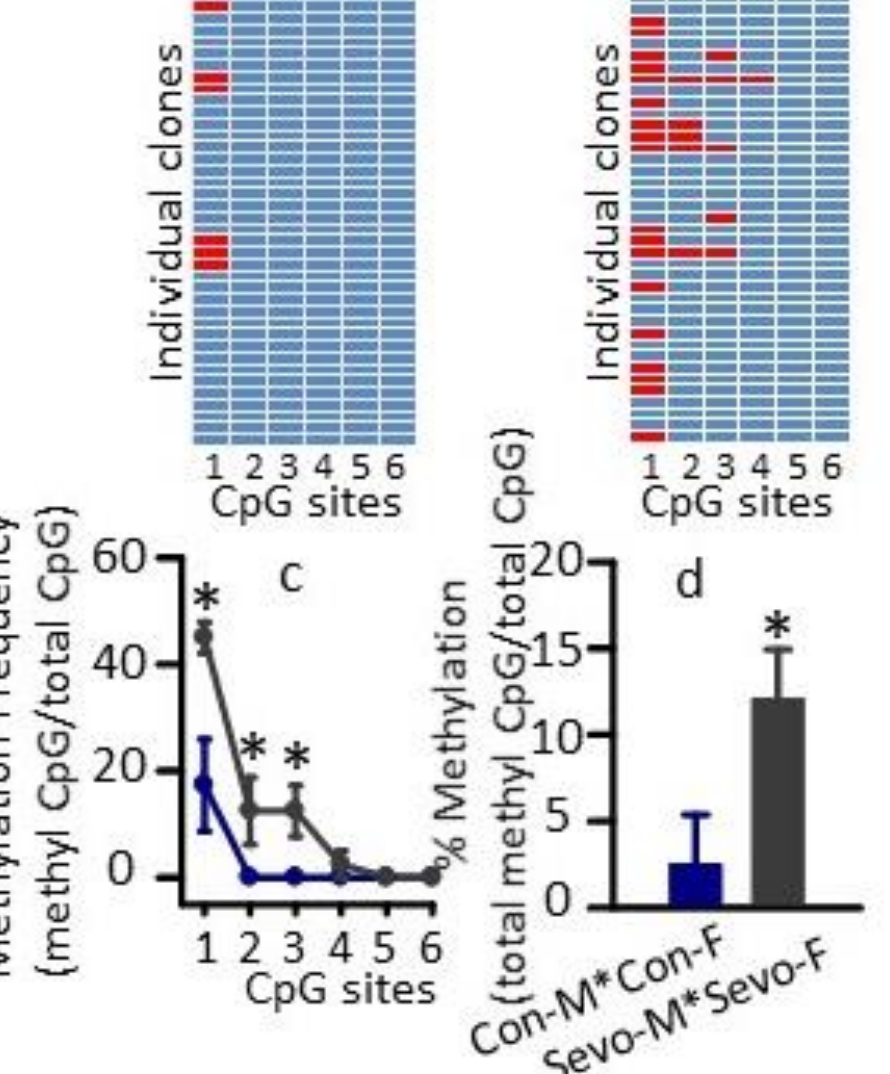

Figure 2. Effects of repeated exposure of young adult rats to sevoflurane on DNA methylation of the Kcc2 gene in sperm and ovary of $\mathrm{G} 1$ exposed rats and in the hypothalamus and hippocampus of their $\mathrm{G} 2$ offspring. Heat maps show distribution of unmethylated (blue cells) and methylated (red cells) CpG sites.
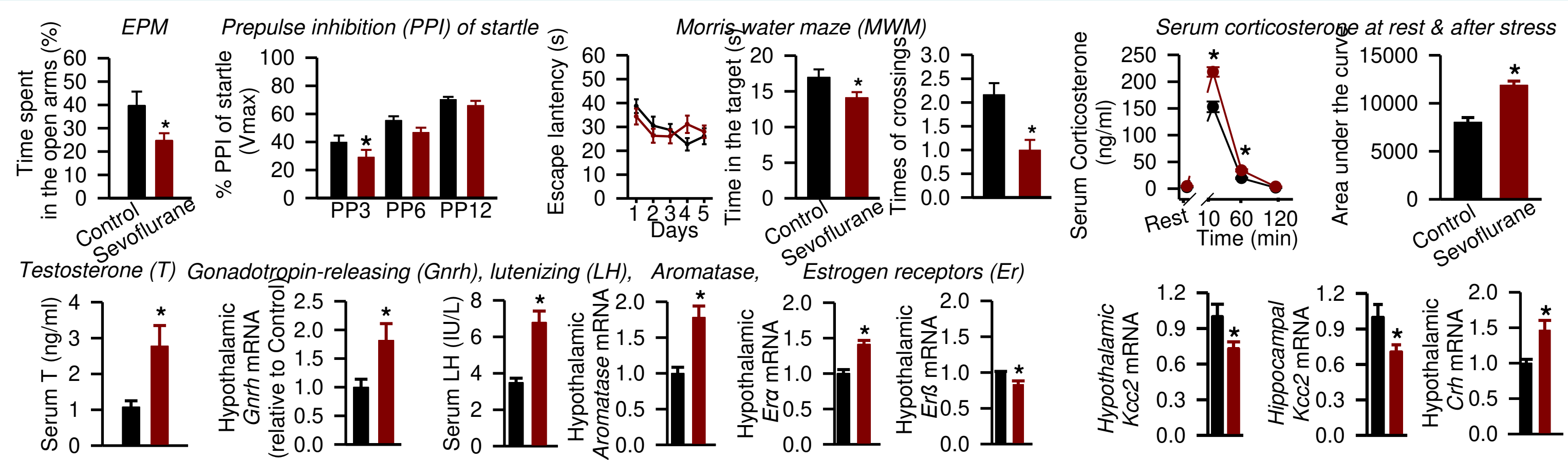

Figure 1. Neurobehavioral and neuroendocrine abnormalities in the exposed $\mathrm{G} 1$ males. Tissue samples were collected on $\geq \mathrm{P} 160$. 